\title{
INTRODUCING MOBILITY: THE MPOLICE PROJECT
}

\author{
Michael Ney ${ }^{1}$, Bernhard Schätz ${ }^{2}$, Joachim Höck ${ }^{3}$, and Christian Salzmann ${ }^{4}$ \\ ${ }^{I}$ Fakultät für Wirtschaftswissenschaften, TU München, Germany; ${ }^{2}$ Fakultät für Informatik, TU \\ München, Germany; ${ }^{3}$ Polizei Oberbayern, München, Germany; ${ }^{4}$ BMW Car IT GmbH, \\ München, Germany
}

\begin{abstract}
Mobile IT is standardly claimed to be a key innovation for modern organizations. But how can this technology be adopted to improve the business processes of an organization? How to assess the benefits of a possible resulting organizational change? How to ensure the adequacy of the technologically driven solution and its adoption? We show how a systematic innovation process can be applied to an organization of the public sector for introducing mobile IT. In the first step, we identify gaps in the business process that can be overcome by mobile IT. In second step, enhanced economic evaluation is used to assess the adequacy of the technical solution. Based on this evaluation, in the final step, suitable technology-driven changes of the organizational business processes are identified.
\end{abstract}

Key words: Mobility, IT, public sector, extended effectiveness analysis, Business Process Analysis, scenario analysis, assessment, process improvement.

\section{INTRODUCTION}

With the growing acceptance of its' "always and everywhere" paradigm mobile computing is one of the most promising approaches in today's IT market. In all sectors of economy and private use, mobile devices like cellular phones, PDAs or notebooks replace traditional media like letters, notepads, etc. Many enterprises use already mobile computing to improve their business processes: Parcel Services use mobile technology to integrate their field staff.

To get from bare potentials of new technologies to the realization and use of innovations Rogers points out, that we have to realize the effectiveness of 
new technologies: As shown in other domains, the impact on work plans and organization as well on future rate of adoption depends on "(...) a combination of an innovation's profitability plus its observability (...)" [Rog95], i.e. economic efficiency. In this paper we focus on the police: To achieve its central function - to serve and protect - the police is depending on the mobility of its units. Therefore mobile communication is an essential functionality - using mobile radio for the support of officers on site. Since the police is a non profit organization (with non profit targets i.e. safety for society) the possible impact is even harder to assess.

While mobile technology has evolved rapidly in the past years, the research of the potential of possible application in coherence with economic efficiency of investments has not kept up. When the Police of Upper Bavaria was introducing mobile IT, its organization was facing the challenges of analyzing and evaluating the benefits of mobile services:

- How does mobile computing possibly influence the work of the police, on the level of the workplace as much as on an organizational level?

- How does it pay off, in terms of the different stakeholders (the officer, the management, and even the society)?

Therefore the task force mPolice was established as a joint project between the Police of Upper Bavaria, Siemens Business Services, and the TU München: The main targets of mPolice is a holistic analysis addressing IT as well as economical issues: On the one hand, to establish the influence of mobile IT on the business processes of an organization from the Computer Science point of view to get a picture of its possible influence; and, on the other hand, to get an understanding of the possible benefits (or draw-backs) for all stakeholders involved from the economical point of view.

In the following we present the results of an enhanced economic evaluation approach, which supports the multilayered task of evaluating mobile services tailored for the specific needs of the police. A set of business processes of the police was analyzed for applicability of mobile computing. After the technical analysis a system was proposed improving these processes. To measure the benefits of an approach, we used the integrated approach of the enhanced economic evaluation. The remainder of this article is structured as follows: In the following section we describe the domain of application, the police. We explain some of the processes as they are practiced. We then explain the methodology to detect the gaps within theses business processes, followed by the methodology to measure the benefits of a possible solution for the public sector. In the final section we present the technical solution we proposed and found it on the expected social and economic benefits. We close with a conclusion that sketches the next steps that follow the results. 


\section{CONTEXT: MOBILE IT AND THE PUBLIC SECTOR}

Upper Bavaria with almost four million inhabitants is the largest operational area of the police within Bavaria. The tasks handled by the police reach from shop lifting to murder, plane crashes, and train accidents. Surely the use of mobile end devices could simplify the work of the police considerably. However, the differences between optimizing in the public sector and private sector have to be considered. The goal of an enterprise is normally to make the most profit of the invested capital. So a most important goal must be to lower the costs to raise the efficiency. Police work however isn't profit orientated. Its goal consists of services such as the recording of traffic accidents, to avert, to prevent, and guarantee general safety in the population. The most important goal is to improve the quality of the services of the police, for example to catch an escaped person quicker or to patrol a critical area with more coverage. Obviously, it is hard to measure the benefits of prevented crimes with the same optimization measures as you do in case of a private enterprise.

\subsection{Application Domain: The police of Upper Bavaria}

Each year the statistics of crime and the clarification quota are published by the Bavarian Police Department. The number of crimes in Bavaria, in comparison with the preceding year, rose by 8.100 to 401.380 or by $2.1 \%$, and in Upper Bavaria by 1.641 crimes to the value of 72.880 . I.e. despite of the rise of crimes the number of clarified cases has risen. This is surprising because the number of public employees of the Bavarian Police Department has hardly changed in comparison with the preceding year. Apart from the high motivation of Bavarian officers, the increasing accouterments of technology have a great effect. More than 15 years ago, all operations were managed manually, using typewrites. The Bavarian Police Department now has an electronic integration method based on the principle of singular recording. The officer records all data at his disposal only once into the databank and can use it to create complaints or notices of accidents according to the requirements. Also the management of operations is largely automated. Thus the handling time for a complaint has reduced from 74 minutes to 24 minutes. However, this case handling is only possible from one Net-PC in the headquarters. Thereby capacities for prevention and enforced investigation were gained. Two thirds of approximately 33.000 employees of the Bavarian Police Department do shift work, which, in $60 \%$ cases, takes place outside of headquarters. Here there are two different forms of field service. In most cases motorized patrols in both civil and uniformed 
vehicles. Momentarily the Bavarian Police Department has 9.000 vehicles in use. The Police Department in Upper Bavaria has approximately 500 uniformed and 650 neutral vehicles at its disposal. In patrol duty the police officers only use a 4m-radio unit, which is part of the vehicle to communicate with headquarters or operation base and with other patrol vehicles. For close-up range to headquarters there is also a $2 \mathrm{~m}$-radio unit. In few exceptions the officers additionally carry a cellular phone. At present in whole Bavaria there are additional 400 mobile tracing clients, which access the Central Computer of the Bavarian LKA (regional bureau of crime investigation) over the GPRS-Network of Dt. Telekom, to enquire tracing data. Presently 1.000 .000 persons and affairs per month are checked over these clients. Furthermore data, which is filed on the central computer, e.g. formats of driver's licenses, forging characteristics of ID papers or car documentation, can be enquired.

However these tracing clients who are built into the vehicles cannot be used for case handling of operations. As the majority of police operations take place outside of the vehicle, the officers only dispose of the $2 \mathrm{~m}$-radio unit, which only has a limited range. Thus the question for the use of mobile end devices for the support of the officers on duty automatically arises. As such, in which sectors and operations is this support possible? As an example we would like to mention the recording of accident notices and the deployment of a hundred Bavarian police officers for the search of a forest area for a searched person. Should an accident occur in duty range of a police officer, more patrols, according to the severity of the accident, are ordered to the place of accident? During the trip the officers receive first information by radio about number of injured or casualties, whether emergency physician has been called, whether the fire brigade is necessary to salvage injured. After the arrival of the crew the rescue of human life resp. the treatment of the injured is first priority. Only after that the actual police work is commenced, e.g. hearing of witnesses, recording of the vehicle data and the checking particulars of the involved, measurement of the site of accident. This data has to be, like in the old days, noted on paper by the officers because there aren't any electronic aids at their disposal. Also the check of the received particulars and the data of the identity papers and car documentation can only be done by radio. Unfortunately this is interfered with other prior radio transmissions and comprises the danger of transmission errors.

\subsection{Structured Approach towards Innovation}

As described in the previous subsections, experiences concerning the introduction of mobile IT gained in other organizations do not readily carry 
over to public organization like the police due to essential differences concerning their organizational and operational structure and goal; nevertheless, adapting mobile IT innovations seemed to be a promising possibility in both domains. However, it remained largely unclear, how innovation might effect the organization, how much benefit could be expected from those innovations, and finally, which possible innovations should be adapted on to ensure maximum benefit. Therefore, a structured, transparent approach to assessing the possible impacts of innovation was needed. To avoid erratic guesses about the impact of the technology, a structured approach is needed that does not leave out important possibilities of improvement by mobile IT. To this end, a structured Business Process Analysis approach especially tailored towards mobile IT as describe in Section 3 is used. Furthermore, to ensure the adaptation by the user at the workplace, to help the management to decide on possible alternatives and necessary organizational changes, and to quantify the payback on the society, a structured and reproducible approach is needed to assess the benefits of the innovation for all stakeholders in a transparent and communicatable manner. To this end, multi-layered economical efficiency analysis as describe in section 4 is applied.

\section{DETECTING THE GAPS: ANALYZING PROCESSES}

To assess the disadvantages and advantages that IT innovation can have for the organization under investigation, we first have to get a clear understanding where new IT technologies will affect the organization most. In the following subsections we explain how those leverage points can be detected in a methodical fashion, by introducing related approaches in Subsection 3.1, show how those approaches can be adapted to mobile IT infrastructure in Subsection 3.2, and finally give a few short examples in Subsection 3.3.

\subsection{Related Approaches from Computer Science}

From the technical point of view, we use Business Process Analysis to analyze where IT innovation can influence the workflow of the organization. Our approach to identifying process improvement is similar to the complete Business Reengineering approach as suggested, e.g., by [HC94]. Like in the approach used there, we use a structured analysis of the existing processes concerning using IT as driving force of change. However, in contrast to 
complete reengineering, we are rather interested in a stepwise improvement of the business process. As explained in Section 2, this is mainly due to the fact that organization of the public sector are not driven by the main factors customer, competition, and change as ,much as in the private sector; consequently, radical organizational change is much more likely not to be adapted than in the private sector. Therefore it is much more important to identify the current process as they are "actually performed" in the organization rather than they are "supposed to be performed". Additionally, when describing the actual processes based on the activity profile we also document why these activities are performed in that fashion (especially including the "positive and negative aspects" as perceived by at the workplace). Thus, when analyzing the processes we will start out from a description of the "workplaces" and combine these to form a picture of the actually performed business processes on the "process level" as well as on the "organizational level".

This evaluation of an organization from different points of views (work place level, process level) becomes even more important when assessing the effects of the improvements inspired by an IT-driven analysis of the business process. Consequently, our incremental approach suggested here is similar to Total Quality Management (TQM) [Mad98]; as shown in Section 4 we will also use a structured analysis similar to Quality Function Deployment (QFD) to assess the benefits; however, in our approach we focus on the IT impact as the driving force to direct the change of the business processes. Furthermore, we use a four-level approach to quantify the impacts on the work-place level, the process level, the organization level, and even the society level, as discussed in detail in Section 4. During assessment, especially the information obtained on the workplace level is reused to ensure the adoption of the process by the end-user.

\subsection{Technology-Driven Process Analysis}

As mentioned above, we are a priori not interested in defining completely new business processes or changing the organizational structure. We are much more interested in "systematically" detecting gaps in the processes which can be "closed using mobile IT infrastructure". Since mobile terminals introduce three different aspects of operation not available with classical IT infrastructure ("mobile information processing", "mobile communication", "constant reachability"), we are especially interested in three kinds of gaps that can be overcome using this technology:

1. Gaps caused by "access centralization": Information is only available at certain locations or steps in the process; the infrastructure supports online access only by means of indirection (e.g., by telephone operators). 
2. Gaps caused by "data replication": Information is reproduced at different steps in the process; the infrastructure does not allow reusing the produced information.

3. Gaps caused by "passive processes": Information is only available at request ("information pull"); the infra structure does not actively distribute information to possible clients ("information push")

To obtain a description of the business processes actually performed, as mentioned above the activities as performed on the individual workplaces (e.g., officer in the field, officer in the control room) were described. Gaps in the business processes show up on the level or the processes and on the level of the organization, depending on their type:

- "Process level": Since in a non-mobile environment access is generally centralized, processes carried out in different workplaces have a tendency to show gaps caused by access centralization. Furthermore, since work places tend in a non-mobile environment generally offer different infrastructure, information is reproduced to cover with these differences.

- "Organization level": Since processes often are not integrated on an organizational level, gaps by information replication are introduced between those processes. Even if processes use shared information, information is not actively forwarded, leading to gaps by passive processes.

To analyze the business process where mobile IT infrastructure can be applied, a four-step approach was used: In the first step, the general tasks of the police and the overall business task including the workplace, the processes, and the organization were examined to describe the overall structure of the organization and the main information flows. In the next step, a more in-depth analysis of the actually performed activities was carried out. The purpose of this step is to identify activities accessing or producing information and to analyze whether this information is available on-line (e.g., by means of interface installed in the car, by radio communication, or by telephone) or off-line (e.g., maps, forms); furthermore, the participation of the system in distributing the information was assessed ("information pull" vs. "information push"). In the second step, those activities in the identified business processes requiring access to information were analyzed. For those activities using on-line access to information, the scenarios describing the interaction including the involved parties were defined. For those activities involving off-line information, additionally, the required or produced information was defined. As a part of the scenario, the sources and the sinks of this information were identified. Additionally - for the "information push/pull" analysis - the initiating events for the generation and the use of the information were identified. In the final step, business processes on the process and on the organizational level were 
constructed from the description of those activities by linking the workplace processes based on the described information flow. By evaluating those links in the defined processes, possible gaps where detected and documented.

\subsection{Application Examples}

To describe the relevant processes in sufficient detail for assessing the IT impact, an approach analogue to [JEJ94] was used, however extended to fit the UML description techniques [FS99] as used in the Rational Unified Process [Kru00]. To identify and structure the overall tasks and the general business processes, use cases structured in form of function trees with actors and interactions were used. Once, the main processes were identified, activity diagrams were used to identify the main activities of a process. For those activities accessing or producing information, detailed sequence diagrams describing the interaction and exchanged information were defined. We illustrate the process analysis using the business process "Recoding an accident" as example. This process includes the activities "Dispatching Car", "Checking IDs" and "In-the-Field Protocol". The first requires on-line information using information pull; the second requires access to on-line information; and the third produces off-line information. The analysis of those activities is explained in more detail in the following.

Figure 1 shows the process of checking IDs of a person involved in an accident. The description of this process is obtained by the combination of the description of the work places "Officer" and "Control Room"; the linking data access is "on-line" ID information obtained by demand via radio. According to the isolated view of this workplace in the current, nonoptimized version, after obtaining the ID of the involved person, the officer waits until a radio connection to an operator in the control room is available. After stating his request and identifying himself, he transmits ID information of the involved person to the operator. The operator then checks the transmitted data with the registry via the intranet and relays the obtained information back to the officers. The officer acknowledges the receipt of the data and returns the ID of the person involved. Analyzing the activities of the control room in this workplace-focused view reveals that it only acts as a relay to the registry concerning the information flow of the "Checking Ids" activity. 


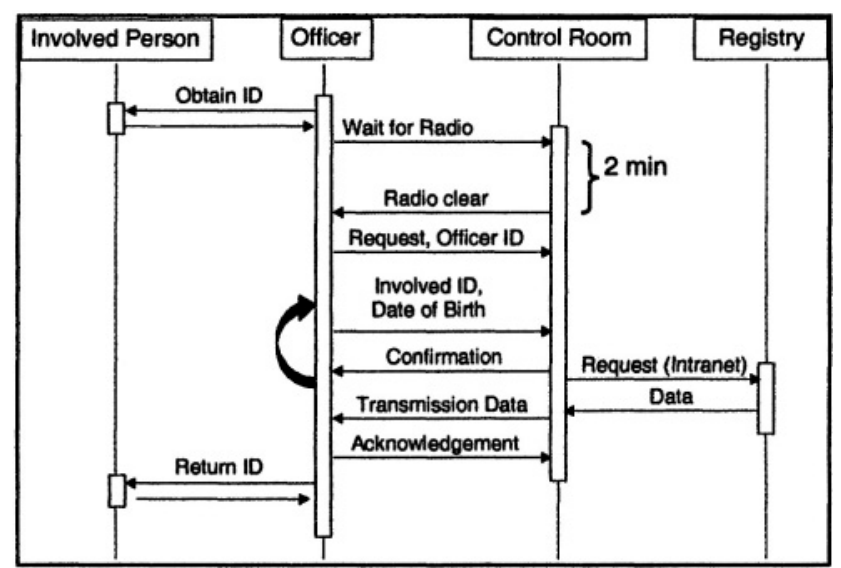

Figure 1. Data Flow for "Controlling ID Papers" (Pre Optimization)

Combing these activities on the process layer by means of their common accessed information (ID data) shows that here a gap caused by centralized access is introduced in the process. As a result, by verbal communication to the operator errors can be introduced and therefore a protocol to avoid those is introduced: information transmitted to the other party is sent back in form of a confirmation; if confirmation and sent data differ, the data is retransmitted (as indicted by the backward loop in Figure 1). Obviously, this gap results in additional delays, either when waiting for an available operator or when retransmitting misunderstood information. Additional to the gap due to data centralization mentioned above we can identify gaps of data replication by linking the activity "In-Field Protocol" to the business process. Once the place of the accident got secured the police officer usually starts to prepare a protocol. During the activity, information is produced in form of hand-written protocols (including the data of the ID check done before. This information is therefore re-entered into the information system upon return to the precinct. Finally, when combining processes on the organizational level, additionally, gaps by passive processes can be identified. During the "Dispatching Car" activity, the control room actively has to obtain information about which available car is in the vicinity of the accident, i.e. query the cars about their position and their status. Only after this information is available, a corresponding car can be assigned to the task.

\section{QUANTIFYING IMPROVEMENTS}

Today many IT projects analyze innovative applications solely orientated on monetary values and isolated from the organizational context. This 
traditional investment calculation process makes sense for many operations side companies. But the police is not operating profit-oriented, but offering public services. Furthermore the traditional calculation makes sense as a prestage for outsourcing, but it's not helpful for introducing innovative work plans and organizational changes. To get an integrated evaluation of mobile solutions, it's not sufficient to analyze the cost effects only on the workplace. An implementation of mobile service in an organization usually has a widely ramified and multi layered impact to many divisions inside and outside an organization.

In the following chapter we introduce the Enhanced Economic Evaluation approach that supports the following options [RHW96, PRW03]: In addition to the consideration of "qualitative" (non monetary) and "quantitative" criteria this approach uses an integrated view of a decision's impact to an organization. The application of the approach is discussed in detail in Section 5.

\subsection{Related Approaches from Business Administration}

To systemize and to point out impacts, our approach uses a multilayer reflection to integrate different point of views into one analysis. This idea originally used in IT and Telecom domain - characterizes a way to get an integrated view on organizational issues [Ant95; Bau96; Bod84; Nie88; PR87; RHW96; RMES00; Zan88] and differs the following layers:

- Layer 1: Workplace: Here we analyze impacts on a specific workplace or workstation that arise from the introduction of mobile services.

- Layer 2: Processes: Here we focus on the effects of the work flow in the organization (e.g. preliminary process steps).

- Layer 3: Organization: On this level we gather primary the value effects of mobile services to the long term efficiency of an organization.

- Layer 4: Society: In this section we analyze the impacts of mobile services on the labor market and the assurance of the location of the industry and economics.

These layers must not be analyzed separately, but tightly linked. They represent different views to the economic actions (i.e. the introduction of mobile services). For this reason we use them as an analytical separation, to point out the correlations and network effects of mobile services: They show i.e. the links between human capital and organization, the process of value creation, the relation between the organization and its environment. Using this multilayer approach we can apply to different views within the police: On layer 1 the policeman, on layer 2 the supervisor and on layer 3 the ministry of interior. The last layer can operationalize the society's impressions of the police i.e. the perceptions of the population. 


\subsection{Integration of Stakeholders}

The utilization of distributed information is the main problem of implementing new technologies in organizations. A large percentage of information - i.e. information regarding the usage of radio communication of the field service - is already available within the organization but distributed in different locations [Ant95, Dek03, PRW03]. Owner of these information are employees in a variety of functions and hierarchies. However, to integrate processes, this information (and above all the availability of this information) becomes increasingly important [RMSE00]. In enhanced economic evaluation the merge of the distributed information proceeds as a moderated group process with the participation of employees from all relevant layers. In order to minimize the risk to adulterate the results it's necessary for the discussion to be moderated by a person outside the organization. Aggravating circumstances are the conventional information flows in the hierarchical structure of the police that handicaps efficient extracting and forwarding of information. The decision quality that can be archived with this approach depends on the consensus of decisions made across the different layers. Therefore the part of the moderator is very important: The quality of the consensus falls if one perception stands up to the rest.

\subsection{Criteria Catalogs}

A basic characteristic of the enhanced economic evaluation is a comprehensive analysis and consideration of the specific situation of an organization. According to recent publications in business administration we deny the option of an objective and universally valid evaluation. Every decision in an organization is related only to a specific situation: The potentials of processes, organizational structure and human resources demand an evaluation approach that considers the "subjective" needs and specific targets of an organization. The enhanced economic value approach uses a criteria catalog to support the evaluation process in an efficient way and to present details of the specific situation clearly showing the decisions, which led to the obtained results. This catalog consists of different basic sets of criteria and can be modified to match the specific needs: i.e. the predefined catalog consists of the 6 basic criteria sets. Due to the safetycritical tasks of the police, the representatives of the police decided to integrate the criteria "safety" into the decision catalog. 


\subsection{Value Benefit Analysis}

For aggregating all criteria containing several layers it turned out to be useful to apply the value benefit analysis: the different criteria get assigned a weight according to their importance to the participants of the workshops. Furthermore, the criteria are linked to a scale that helps to attach the parameter value of criteria to an achievement of objectives (that is aligned to a certain amount of points). To get a integrated evaluation - meeting the specific needs and including the distinctions of an organization - the points of criteria are multiplied with their weight and summed up to get the result of the evaluation.

\section{RESULTS}

From March to August 2002 the Police headquarter Oberbayern started a pilot study in cooperation of the Siemens Business Services and Technische Universität München, to analyze the applicability of mobile services to enhance efficiency of police's processes.

\subsection{Project Steps}

To analyze the current processes of the police, to identify improvements, and to assess the impact, the project included four steps:

$I^{\text {st }}$ "Monitoring Shift Work" to assess the current situation at the Police: In the preliminary stages of mPolice we first had to get an overview about the current process situation. Therefore we attended several work shifts in differrent police departments. As a result of several interviews with police officers we got knowledge about the current process situation and an overview about potential gaps within these processes that might be closed with mobile services

$2^{\text {nd }}$ "Expert Interviews" to survey possible process improvements: After the monitoring of the shift work at the police we focused on the potential gaps within the police processes. As a basic principle it is useful to select participants with different backgrounds and a variety of functions and hierarchies (e.g., k-9, criminal investigation, traffic). Within 15 expert sessions we demonstrated possible mobile solutions (including latest mobile technology) to get a first idea of possible applications. Many officers started to integrate their vision of innovative mobile services. After that we matched the different potentials of mobile services to the potential process gaps. In these expert interviews we primarily focused on concerns police officers 
have using this new technology. In the introductions we focused on advantages that are given by insights to organizational coherences.

$3^{\text {rd }}$ "Focus Group Workshops" to combine the upper results and aggregate them to a criteria catalog: Within the previous process steps we identified dedicated police officers interested in the domain of mobile services. We invited these officers to our focus group workshops. The starting point of these workshops was the development of global targets based on the strategic goal of the police. We used the results of the preanalysis based the preparatory work mentioned above as starting point. The results of step 1 and 2 included a variety of information, like detailed process analysis, software and hardware requirements used by the participants to fix the following global target categories for the evaluation: Even though the "cost" aspect is also important for a non-profit organization, it is obvious that the police doesn't decide only based on monetary values. Thus, the officers in charge attached importance further aspects influenced by mobile technology: The first is to enhance the "safety" of field service by raising the "quality" of the cooperation of field service and the operator. The second is the increase the "flexibility" of Field service in information retrievals. And third, the reductions of throughput time by optimizing the information flow from the field service to the departments. By minimizing redundant work and the through put time several interviews with officers of the police presented the acceptance of the employees (i.e. "human situation") and of the "society" as general condition. By aggregating these results we got a first level catalog including the following dimensions: Human Situation, Costs, Time, Quality, Flexibility and Safety.

We then integrated the three different dimensions: On the first dimension we had to consider the results from the Business Process Analysis (as mentioned in chapter 3) that analyzed the potentials of mobile services by the existing gaps in the process flow. The Business Administration (chapter 4.1) showed us that mobile services had impacts not only on the specific workplace but the whole organization. Finally we had to integrate the specific (hard and soft facts based) strategic goals of the police (as described above). Aggregating these three dimensions we came up to a cube that represents with each dimension one single point of view.

Prepared with this model the participants could discuss different targets and to select relevant suggestions without being afraid of forgetting something. So this discussion established a basis for an integrated view to organizational coherences. The Mobility Cube including the origin, impact and dimension of mobile potentials represents the most important criteria and illustrates the base for the construction of the criteria catalog. It turned out, that participants rated some aspects with a large variation:; i.e., depending on the function of the participant, the rating of global criteria like 
safety was either attached with more or less importance. Finally the participants agreed to the following result regarding the weight of the different dimensions: The most important factors according to the workshops are an increase in safety and quality of cooperation: According to the weight of safety and quality these criteria represent already $46 \%$ of the whole evaluation (23\% each). Including the criteria of enhancements of flexibility (15\%) and improvements of the human situation $(15 \%)$ these factors represent more than $75 \%$ of the result. The feedback of the society as well as impacts in Cost and Time Savings are comparatively unimportant for the evaluation with a weight summed up to less than $25 \%$. Summarizing the results: The participants of several workshops rated the so called soft facts more important than the traditional monetary based hard facts.

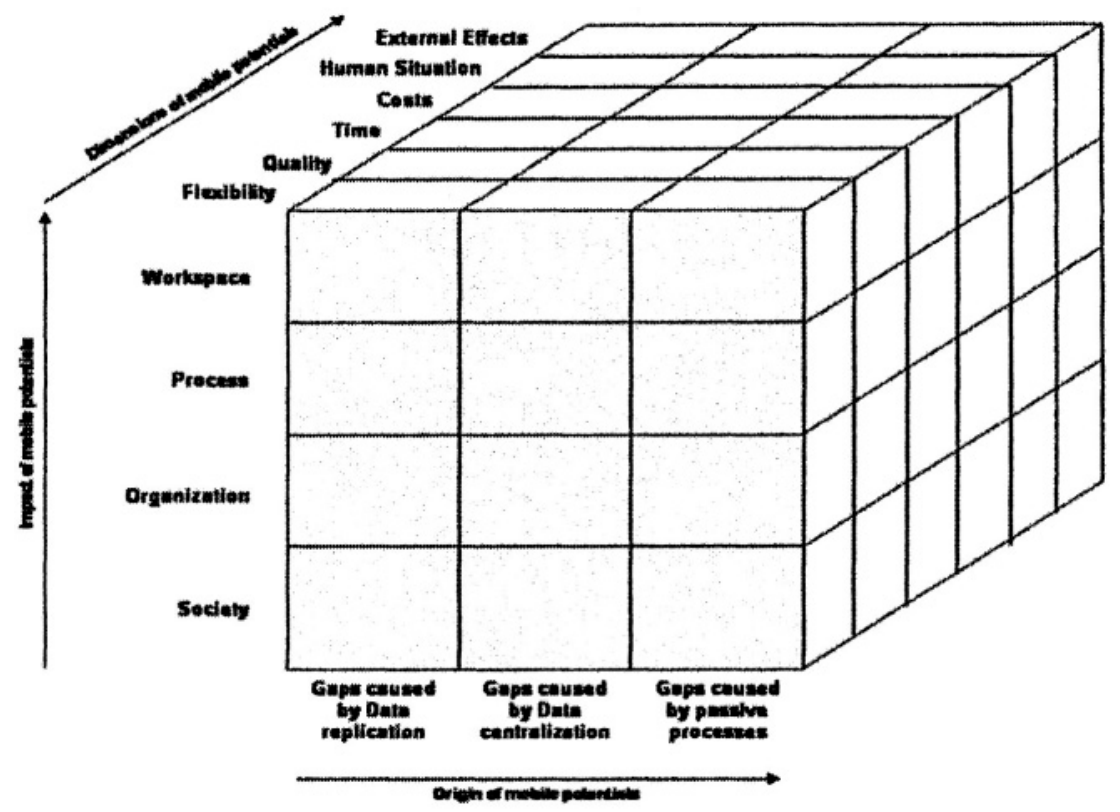

Figure 2. Mobility Cube

$4^{\text {th }}$ "Design and Implementation" of a exemplary analysis of mobile solution's economic efficiency for the police: The cube was not uniformly distributed, i.e. the different potentials of mobile technology affect the organization in different ways:

- On the "work place layer" basically potentials of gaps caused by data centralization have an influence to the field service: In the pilot study we observed significant timesaving originated by the use of a non-limited communication channel (in contrast to the delay with radio communication). Furthermore we noticed the realization of potentials 
caused by data replication: By reducing redundant work (i.e. the information retrieved by the officer in field are reused for the protocol) we assessed an increase in the flexibility and a decrease in the through put time of some processes.

- On the "process layer" we observed advantages caused by the overcoming the gaps of data centralization in conjunction with gaps of data replication. By integrating the field service and department based on the level of information, the information exchange between the central and decentral units gets accelerated: e.g. the information about the possible victims of a traffic accident are available to the operator as soon as the field service retrieved the IDs from the participants.

- On the "organization layer" finally we found mainly potentials by overcoming the gaps of passive processes: By supplying the field service with current information (i.e. push services) the safety of the police officers gets boosted: i.e. by sending accurate information about a menace the infield service can handle situations on a better level of information.

As mentioned above by closing gaps through data replication we get a reduction in through put time that leads to an increase of customer satisfaction. Additionally by overcoming the lacks of data centralization the police can minimize the manual administrative task of information supply and focus on their core tasks.

\subsection{Identifying Solutions}

As described in section 3.3, by the usage of Business Process Analysis we obtain a detailed description of the interactions performed during in-field business processes. By focusing on the activities generating and using information (e.g., accessing a data base, writing a report) and later on identifying classes of coherent information (e.g., accident report), we are able to extract the data flow behind those processes. This method leads to an information centric view instead of a process centric view. Using the information centric view, the interactions from the business processes were used to develop a life history of the different classes of information including its generation, transformation/update, use, and destruction. Using the life histories, gaps in the business process and possible optimizations were identified:

- Gaps caused by access centralization: For process steps accessing centralized information (e.g., terminal-based access to data bases), mobile terminals are used to substitute proxies (e.g., the control room operator). If information is distributed with different access points (e.g., 
different data bases for different crimes), a new access point serving as a common front-end (e.g., common query interface) is added.

- Gaps caused by data replication: For process steps producing off-line information (e.g. accident reports) that are later added manually to the system, a suitable mobile terminal is identified; furthermore, if no online connection is suitable for this interaction, automatic integration of the generated information from the terminal to the system via defined access points (e.g., docking stations) are defined.

- Gaps caused by passive processes: For process steps requiring an active interaction to obtain up-to-date information, the processes are adapted to transfer the responsibility of initiating the update to the producer of the information and to automate the forwarding of updated information to the possible users.

Using these strategies, optimized versions of the business process can be constructed. Using the example of the previous sections, the following optimizations can be applied to close the identified gaps:

1. Checking ID: During "Checking ID", the optimized version using a mobile terminal (e.g., a PDA with GPRS connection) avoids gap introduced by data centralization and therefore leads to a leaner process. Here, after obtain the ID information and performing a local logon to the PDA, the officer enters the information into the PDA and transmits it to the registry via the GPRS connection. Since the information is transmitted as entered by the officer, no cross-checking is required. While the sequence diagrams look similar on first sight (we left out the two-way radio in the first diagram), the optimized process eliminates the delays and iterations introduced by the relay function of the control room. The reduced complexity of the interaction results in a shorter execution time, less possibilities to insert errors, and therefore to improved reliability of performance and less distraction for the officer.

2. In-Field-Protocol: During the "In-Field Protocol" activity, information can be added into a mask and entered online into the system for further editing by the officer once back at the precinct.

3. Dispatching Car: The gap identified in the "Dispatching Car" activity can be closed by adding information push, using a GPS module and remote access to the availability database through the car terminal. By actively supplying this information to the system, the system can identify cars available is in the vicinity of the accident.

Note that these optimizations depend on the available technical infra structure applied. E.g., in case of passive processes, the possible substitution depends on how much information can be generated/forwarded automatically: a car equipped with a GPS module can transfer its location information; by adding a mobile terminal, availability changes can be 
actively announced by the car personnel. Assuming different possible mobile IT infra structures, accordingly different possible optimized processes can be defined. However, the optimized business processes describe a system that is optimized with respect to the "functional data flow" of the organization. For the adaptability of this mission critical solution, "non-functional aspects" (like direct and indirect costs of ownership, change in the organizational structure), of the solution often are of even bigger impact then the functional aspects. As mentioned above, using the extended efficiency analysis, we obtain a metrics to quantify the impact of a solution on the individual as well as on the organizational level by including different aspects like cost, time and quality, as well as flexibility and personal situation, or public appearance and security.

To quantify these different factors, the enhanced economic evaluation is used [RHW96]. To apply this technique, different improvements are described in form of configurations. The definition of a configuration includes:

1. Selecting processes to be substituted and substituting processes (since in different configurations different organizational changes are assessed)

2. Identifying the necessary IT infrastructure (possibly fixing possible variants like bandwidth, reaction time, size of display)

3. Developing usage profiles (number of users, number of request, amount of data, etc.)

Using these configurations, a possible optimized system can be described in sufficient detail to have it evaluated using the enhanced economic evaluation. Accordingly, business process optimized with respect to information flow may show drawback concerning other evaluation aspects, leading to a reduced overall result. For example, the optimized access to the ID database introduced in section 3 has several different consequences:

- Positive factors:

- "Flexibility": Access to data base is independent of availability of operator in control room

- "Quality": Possible errors in the transmission are minimized

- "Time": Time to obtain information is reduced

- "Public appearance": Minimized interaction time with involved parties and higher quality of response lead to better acceptance

- Negative factors:

- "Security": Distraction (loss of eye contact when switching from radio to terminal), loss of surveillance (operator in control room as third party)

Thus, defining different configurations and applying the metrics of the enhanced economic evaluation to each of them can be used to identify the overall optimized solution to the problem. Accordingly, the method can be 
applied to pre-estimate the influences of technological impacts and increase the possibility of adoption by decision-makers as well as users, leading to improved system acceptance.

\subsection{Quantified Expectations}

To illustrate the results, we use a limited mobility cube, reducing the dimensions to costs and quality. [RNW02]. In the following we exemplify an evaluation on a fictive mobile system. Therefore we analyze two alternatives of mobile services: The first alternative contains the latest and current mobile hardware using software developed for the mass market. This system represents - due to the only minor differences in design - an economically priced system with a large range of functionalities that is limited to enhancements. The second alternative consists of a mobile system specially developed for the police (based on a Business Process Analysis): e.g. the migration of the existing functionalities of the Car PC to mobile devices. The hardware offers lower performance but is provably reliable. As a consequence to the special development for the police this system is high priced and comes with limited functionalities (but due to the open software architecture open for enhancements).

Figure 3 shows the results for the costs criterion. The first alternative performs better than the second one due to the costs for the acquisition and the maintenance for the system. Due to the extra design and development of this system it is obvious, that alternative 2 costs more than the first one only composed of mass market hardware.

\begin{tabular}{|c|c|c|c|c|c|}
\hline & & \multicolumn{2}{|c|}{ Alternative 1} & \multicolumn{2}{|c|}{ Alternative 2} \\
\hline Criteria & Weight & Score & Sum & Score & Sum \\
\hline \multicolumn{6}{|l|}{ Costs } \\
\hline planning reliability & $58.33 \%$ & 0 & 0.00 & 0 & 0.00 \\
\hline Costs for Training & $13.89 \%$ & -1 & -0.01 & 2 & 0.02 \\
\hline Costs for Acquisition & $12.22 \%$ & 3 & 0.03 & .5 & .0 .05 \\
\hline Costs for Maintenance & $15.56 \%$ & 3 & 0.04 & .2 & -0.03 \\
\hline Sum & $100.00 \%$ & & 0.06 & & -0.06 \\
\hline
\end{tabular}

Figure 3. Exemplary evaluation on a fictive mobile system: Costs

Concerning quality we can observe a different result (see figure 4): As a result of the used mass market conform standard software we observe that the introduction of mobile services doesn't increase the information level for the $\mathbf{1}^{\text {st }}$ alternative. In contrast the use of the $2^{\text {nd }}$ alternative - including the migration of known functionalities from the Car PC - increases the information level. Additionally, the willingness to adopt the second alternative is much higher, because it is based on an established system. Thus the preparatory training is limited to handle the mobile hardware in 
contrast to the first alternative that takes an extended training including the use of the new software. But even after an adaptation phase the system alternative 1 differs by the limitation of functionalities that can't be enhanced.

As mentioned in chapter 5.1 the criteria quality plays a part of $23 \%$ and costs $8 \%$. Aggregating the results of the exemplary evaluation of the fictive mobile system, we get a surprising result: Summing up the results in quality the score of the first alternative is -0.20 and the score of the second one is + 0.26 . Even though the hard facts seem to be promising for the $\mathbf{1}^{\text {st }}$ alternative, the so called soft facts determine the result: The second alternative - even though it's more expensive and equipped with less functions reaches a better evaluation than the first alternative.

\begin{tabular}{|c|c|c|c|c|c|}
\hline \multirow{2}{*}{ Criteria } & \multicolumn{3}{|c|}{ Alternative 1} & \multicolumn{2}{|c|}{ Alternative 2} \\
\hline & Weight & Score & Sum & Score & Sum \\
\hline \multicolumn{6}{|l|}{ Quality } \\
\hline Qualification of Employees & $9.67 \%$ & 0.00 & 0.00 & 0.00 & 0.00 \\
\hline Lovel of Information & $11.33 \%$ & -4.00 & -0.10 & 4.00 & 0.10 \\
\hline Avallablity of Extern Know How & $11.33 \%$ & 1.00 & 0.02 & 2.00 & 0.05 \\
\hline Enhancement with additional functionalities & $14.67 \%$ & -3.00 & -0.10 & 3.00 & 0.10 \\
\hline Error ratio on the man/machine interface & $8.00 \%$ & -3.00 & -0.05 & 2.00 & 0.03 \\
\hline \multicolumn{6}{|l|}{ Conditions of Employment } \\
\hline Usability & $16.11 \%$ & 0.00 & 0.00 & 0.00 & 0.00 \\
\hline Robustness of hardware & $16.11 \%$ & 0.00 & 0.00 & 1.00 & 0.03 \\
\hline Reliability of the software & $12.78 \%$ & -2.00 & -0.06 & 2.00 & 0.06 \\
\hline Summe & $100.00 \%$ & & -0.29 & & 0.37 \\
\hline
\end{tabular}

Figure 4. Exemplary evaluation on a fictive mobile system: Quality

\section{RELATED WORK}

The possibilities of mobile information technology with respect to improvements of the workplace, its role in improving overall business processes, and its forms of impact have been studied before:

- [BB96] shows the two decisive factors of mobile technology for supporting the workflow: mobility to support access to share resources ("immediate access"), as well as mobility to be available at any time ("constant reachability"). However, in contrast to our approach, they focus on a common (virtual) workspace with immediate interaction of the participants and the collaborative aspect rather than considering individual business processes.

- [LH98] points out that the central aspect of mobility in most work places is not so much the immediate interaction of all participants but much more the integration of the individual interactions performed at each workplace to support a non-interrupted workflow of the participants. In contrast to our approach, however, they do not use an explicit model of the workflow to discover gaps in the business processes performed. 
- [KS02] shows the consequences of the always access/always on paradigm by introducing three aspects of mobility: The possibility to receive the necessary information on the spot of activity ("spatial mobility"), the possibility to get immediate response ("temporal mobility") and to support context awareness ("contextual mobility"). However, while they focus on the classification, we focus on using these aspects to identify gaps in business processes and propose solutions to overcome those gaps.

In general, while those approaches discuss fundamental aspects of integrating mobile technology in the workflow, we concentrate on the application of those aspects to create innovative business processes: The main focus of our approach lies on "systematically" analyzing business processes to identify possible innovations that can be realized using mobile IT and that are likely to be accepted by the users. By combining Business Process Analysis (BPA) and Enhanced Economic Evaluation (EEE) we ensure that a balanced solution considering optimization with respect to workflow as well as with respect to socio-economical factors is identified.

\section{CONCLUSION}

In this paper we presented our experiences in analyzing the options of mobile computing in a concrete application domain. We presented a way to determine the benefits of such an application, tailored specially for the needs of the public sector, including the points of view from computer science and business administration. The Enhanced Economic Evaluation (EEE) assists the introduction of innovative mobile services in cooperation with the employees as an incremental innovation, particularly with respect to the nonquantitative goals of non-profit. By adapting the criteria weights of the EEE, the approach presented here can also be adapted to the needs of organizations of the private sector.

As mentioned, the deployment of the enhanced economic evaluation approach aims on different perspectives: The computer science with its Business Process Analysis helps to analyze media breaks within a process or between processes (gaps caused by passive processes, access centralization, and data replication) and to suggest solutions using mobile infrastructure effectiveness. But this increase of effectiveness doesn't implicate an economical surplus of an innovation: Besides optimization of the process (i.e. reduction of media breaks and improvements to the different analyzed gaps) organizations have to make sure that they get to know the impact of an innovation to the established organization (on processes, organization,) and society, and their related aspects (e.g., security, human situation). 
In the domain of organizational mobile services there's a change in innovation efforts towards the organization - employee interface to establish successful mobile business models. Therefore the computer science as well as the business administration perspective has to develop suitable approaches and techniques to tie an integrated evaluation system to the implementation of innovations. The instruments of the Business Process Analysis combined with Enhanced Economic Evaluation seem to be promising approach for this purpose.

\section{REFERENCES}

[Ant95] Antweiler, J. Wirtschaftlichkeitsanalyse von Informations- und Kommunikationssystemen, Datakontext, 1995.

[Bau96] Bauer, R. Die Methodik der Erweiterten Wirtschaftlichkeitsbetrachtung, in: Reichwald, R Henning, K. FAMO, Augustinus, 1995.

[BB96] Bellotti, V. and Bly, S. Walking away from the desktop computer: Distributed collaboration and mobility in a product design team. Proceedings of the CSCW '96, Cambridge, MA, 1996, pp. 209-218.

[Bod84] Kommunikationstechnik und Wirtschaftlichkeit, München 1984.

[Dek03] Deking, I. Management des Intellectual Capital, Gabler 2003.

[HC93] Hammer, M., Chamy, I. Reengineering the Cooperation: A Manifesto for Business Revolution. Harper Collins, 1993.

[JEJ94] Jacobson, I., Ericsson, M., Jacobson, A. The Object Advantage - Business Process reengineering with Object technology. Addison Wesley, 1994.

[FS99] Fowler, M. Scott, K. UML Distilled. Addison Wesley, 1999.

[Kru00] Kruchten, P. The Rational Unified Process. Addison Wesley Series, 1999.

[KS02] Kakihara, M. and Sorensen, C. Mobiliy: An extended perspective. Proceedings of the 35th Hawaii International Conference on System Sciences, 2002

[Mad98] Madu, C. (ed.) Handbook of Total Quality Management Kluwer Academic Publishers, 1998.

[LH98] Luff, P. and Heath, C. Mobility in Collaboration. Proceedings of the CSCW '98, Seattle, WA, 1998, pp. 305-314.

[Nie88] Konzepte der Wirtschaftlichkeitsberechnung bei integrierten Informationssystemen, in Horvath, P Wirtschaftlichkeit neuer Produktions- und Informationstechnologien, Schäfer-Poeschel, 1988.

[PR87] Picot, A. Reichwald, R. Bürokommunikation, 3rd Edition, Hallbergmoos, 1987.

[PRW03] Picot, A. Reichwald, R. Wigand, U. Die grenzenlose Unternehmung, 2003.

[Rog95] Rogers, E. The Diffusion of Innovation, Berkeley, 1996.

[RHW96]Reichwald, R. Höfer, C. Weichselbaumer, J. Erfolg von Reorganisationsprozessen, Schäfer-Poeschel, 1996.

[RMSE00] Reichwald, R. Möslein, K. Sachenbacher, H. Englberger, H Telekooperation, 2nd Edition, Springer, 2000.

[RNW02]Reichwald, R Ney, M Wagner, M. Kundenintegrierte Entwicklung von mobilen

Diensten, in: Reichwald, R. Mobile Kommunikation, Gabler 2002, p. 347 - 359.

[Zan88] Zangl, H. CIM Konzepte und Wirtschaftlichkeit, in OM, $36 \mathrm{Jg}$. (1988). 